\title{
Metaplastic carcinoma of the breast: Imaging and pathological features
}

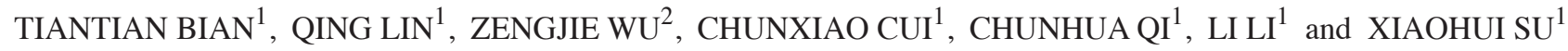 \\ ${ }^{1}$ Department of Breast Imaging and Radiology, The Affiliated Hospital of Qingdao University, Qingdao, \\ Shandong 266100; ${ }^{2}$ Department of PET/CT, Qingdao Center Medical Group, Qingdao, Shandong 266042, P.R. China
}

Received February 6, 2015; Accepted December 21, 2015

DOI: $10.3892 / \mathrm{ol} .2016 .5177$

\begin{abstract}
Metaplastic carcinoma of the breast is a rare form of breast cancer. The aim of the present study was to investigate the imaging and pathological features of metaplastic carcinoma. The features identified on mammography and sonography were retrospectively reviewed in 13 women with metaplastic carcinoma of the breast. The results from the mammographs and sonographs were additionally evaluated using immunohistochemical staining for the expression of the estrogen receptor (ER) and progesterone receptor (PR), receptor tyrosine-protein kinase erbB-2 (CerbB-2) and P53. The most common features observed in the mammographs were as follows: Irregular and oval shaped masses, 53.8 and $46.2 \%$, respectively; spiculated and circumscribed margins, each $30.8 \%$; and high and marginally high density masses, 69.2 and $30.8 \%$, respectively. The most common sonography features observed were as follows: Hypoechoic masses, 84.6\%; complex echogenicity, 76.9\%; irregular, round and oval shaped masses, $69.2,30.8$ and $30.8 \%$, respectively; indistinct and circumscribed margins, 53.8 and $46.2 \%$, respectively; an abundant blood flow, $53.8 \%$; and posterior acoustic enhancement, $61.5 \%$. The immunohistochemical (IHC) profile for 13 patients demonstrated that ER was not expressed in $100 \%$ of patients, PR and CerbB-2 were not present in $92.3 \%$ of patients, and P53 was present in $63.6 \%$ of patients. Therefore, metaplastic carcinoma of the breast exhibits more benign IHC features compared with invasive ductal carcinoma. In addition, it may be challenging to diagnose patients that do not possess posterior acoustic enhancement or express hormone receptors from other types of breast cancer.
\end{abstract}

Correspondence to: Professor Qing Lin or Miss. Tiantian Bian, Department of Breast Imaging and Radiology, The Affiliated Hospital of Qingdao University, 59 Haier Road, Qingdao, Shandong 266100, P.R. China

Email: linqing9180@hotmail.com

Email: tiantianbian5001@163.com

Key words: breast neoplasm, metaplastic breast carcinoma, mammography, color doppler flow imaging, pathology

\section{Introduction}

Metaplastic carcinoma of the breast is a rare form of breast cancer and possesses a poorer prognosis than other common types of breast cancer (1). Metaplastic carcinoma exhibits the metaplastic transformation of glandular epithelium to squamous epithelium and mesenchymal tissue, and accounts for $<5 \%$ of all breast cancers (2) and may contain glandular and non-glandular components with mixed epithelial and mesenchymal tissue. Involvement of the axillary lymph node is rare, with hematogenous metastasis occurring more commonly in cases of metaplastic carcinoma $(1,3)$. Metaplastic carcinoma demonstrates a poorer prognosis and a higher risk of recurrence compared with other types of breast cancer (3). It typically presents as triple negative, therefore the effects of hormonotherapy are limited $(3,4)$. Due to the heterogeneity of metaplastic carcinoma and its increased complexity compared with more common types of breast cancer, mixed chemotherapy regimens and dose schedules may be more effective and appropriate (2). Due to the distinction in surgical treatment and chemotherapy between metaplastic carcinoma and other breast cancers, it is particularly important to diagnose the tumor correctly (2). Metaplastic carcinoma demonstrates a number of benign features similar to invasive ductal carcinoma in mammograms and sonograms, and subsequently may be misdiagnosed as benign lesions (4). Therefore, the pre-operative differential diagnosis is particularly important as each form of cancer warrants specific management. Although there have been numerous clinical and pathological features reported regarding metaplastic carcinoma, to the best of our knowledge, few imaging features have been described (5). Therefore, the aim of the present study is to demonstrate imaging features of metaplastic carcinoma of the breast and associate these with pathological and immunohistochemical results.

\section{Materials and methods}

Patient characteristics. The present study retrospectively reviewed data collected from 15 patients with metaplastic carcinoma of the breast treated at The Affiliated Hospital of Qingdao University (Qingdao, China) between March 2012 and May 2014. In total, 2 cases were excluded from the review 
of imaging features due to a lack of mammography and sonography images; therefore, 13 patients were retrospectively reviewed for mammography and sonography features. Immunohistochemical results were reviewed for all 15 patients. The patients were aged 37-80 years (mean, 49 years). All the patients originally presented with a palpable mass in the breast, the duration of which varied between 15 days and 1 year.

Mammographic examination and image analysis. Mediolateral-oblique and craniocaudal images of each breast were obtained with standard compression using the Selenia ${ }^{\circledR}$ Dimensions ${ }^{\circledR}$ Mammography System (Hologic, Inc., Marlborough, MA, USA). The mammography images were retrospectively reviewed by 2 radiologists with $>5$ years individual experience of breast imaging. The 2013 American College of Radiology (ACR) breast imaging reporting and data system lexicon (BI-RADS) was used to review the images (6), as follows: The shape of the lesion may be classified as round, oval, lobulated or irregular; the margin surrounding the lesion may be classified as circumscribed ( $>75 \%$ of the margin is well defined), micro-lobulated, obscured indistinct or spiculated; and the density of the lesion may be classified as high, iso or low.

Sonographic examination and image analysis. The ultrasound images of each breast were obtained using a Siemens Acuson S2000 and EBU 7500 10-14 MHz linear probe (Siemens AG, Munich, Germany). The sonography images were retrospectively reviewed by 2 radiologists with $>8$ years individual experience in breast sonography imaging, according to the 2013 ACR BI-RADS lexicon, and were distinguished by shape, margin, boundary, echo pattern and the posterior echo feature. Color Doppler flow imaging was used to observe the shape and distribution of blood flow in and surrounding the mass.

Histopathology. Histopathological results were obtained following surgical resection. A pathological examination was performed for all formalin-fixed (Tieta; Laiyang Far East Barrel Manufacturing Co., Ltd.), paraffin-embedded (Taicang City Haotian Technology Co., Ltd., Suzhou, China) and hematoxylin-eosin (LABEST, Beijing, China) stained representative tumor samples (3-5 $\mu \mathrm{m}$ thickness). A light microscope with a $\mathrm{x} 40$ objective lens (Olympus BX43; lens, CH-BI45-T; Olympus, Tokyo, Japan) was used to observe tumor tissues. Immunohistochemical (IHC) staining (SuperPolymer, Shanghai, China) and chromogenic in situ hybridization staining (ZytoDot ${ }^{\circledR}$ SPEC HER2 Probe Digoxigenin; ZytoVision GmbH, Bremerhaven, Germany) for estrogen receptor (ER), progesterone receptor (PR) and receptor tyrosine-protein kinase erbB-2 (CerbB-2) (OriGene Technologies, Inc., Beijing, China) were performed using the conventional peroxidase, anti-peroxidase detection method, and peroxidase-labeled streptomycin resistance biotin staining was used for IHC staining. Positive staining for ER and PR was defined based on the percentage of stained nuclei, and CerB-2 status was evaluated based on the percentage of tumor cells and the intensity of membrane staining. The scores of HER2/CerB-2 varied between 0 and 3+, depending on the method recommended for the DakoHercep Test (Dako Denmark A/S, Glostrup, Denmark). Tumors with scores of 3, or with a $>2.2$-fold increase in HER2 gene amplification, as determined by fluorescence in situ hybridization (ZytoLight ${ }^{\circledR}$ SPEC HER2/CEN 17 Dual Color Probe; ZytoVision GmbH) were considered to be positive for HER2/CerB-2 overexpression.

The histopathology images were retrospectively reviewed by 2 pathologists each with $>5$ years individual experience in the pathological diagnosis of breast diseases. IHC investigations for the presence of the ER and PR, CerbB-2 and P53 were performed on all masses, and axillary lymph node involvement was assessed by pathological diagnosis.

\section{Results}

Pathological findings. Palpable masses in unilateral breasts were observed in 13 female patients (median age, 49 years; age range $37-80$ years). In total, 8 masses were identified in the left breast and 5 in the right breast. The diameter of the masses ranged between 0.9 and $6 \mathrm{~cm}$.

Mammography images were available for 13 patients. In total, $84.6 \%$ had dense breasts. The mammographic features are revealed in Table I. A representative mammography scan is presented in Fig. 1A. The mammographic features observed in the 13 patients were as follows: 7 patients $(53.8 \%)$ possessed irregularly shaped masses, while 6 patients $(46.2 \%)$ possessed round or oval-shaped masses; circumscribed margins and spicules were each identified in 4 patients; high density masses were detected in 9 patients $(69.2 \%)$, while 4 patients $(30.8 \%)$ possessed iso-density masses; and micro-calcifications were identified in 1 patient $(7.7 \%)$.

Sonography findings. The sonographic findings are revealed in Table II. Representative sonograph images are shown in Fig. 1B and C. The sonographic features observed in the 13 patients were as follows: Irregularly shaped masses were detected in 9 patients $(69.2 \%)$, while 4 patients $(30.8 \%)$ possessed round or oval masses; 6 patients $(46.2 \%)$ possessed circumscribed margins, while spicules were detected in 7 patients (53.8\%); 10 patients $(76.9 \%)$ demonstrated complex echogenicity, while 3 patients $(23.1 \%)$ demonstrated homogeneous echogenicity (complex echogenicity consists of a predominantly solid mass with certain tiny cystic regions throughout); posterior acoustic enhancement was detected in 8 patients $(61.5 \%)$; abundant blood flow was identified in 7 patients $(53.8 \%)$, while a low blood flow was detected in 3 patients $(23.1 \%)$; and little blood flow was detected in 3 patients $(23.1 \%)$.

Final pathological diagnosis. The final pathological diagnoses were obtained by surgical biopsy for all 15 patients. In total, 8 patients were diagnosed with squamous cell carcinoma, 3 patients with spindle cell carcinoma, 2 patients with matrix-producing carcinoma, 1 patient with fibromatosis-like carcinoma and 1 patient with mixed metaplastic carcinoma. The overall immunohistochemical profile for all 15 patients is demonstrated in Table III. Briefly, 13 patients (86.7\%; 13/15) did not express ER, 14 patients $(93.3 \%$; 14/15) did not express either PR or CerbB-2 and 7 patients $(63.6 \% ; 7 / 11)$ did express P53. Axillary lymph node involvement was observed in 1 patient. 
Table I. Mammography features and tumor types of 13 patients with breast metaplastic carcinomas.

\begin{tabular}{|c|c|c|c|c|c|}
\hline \multirow{2}{*}{$\begin{array}{l}\text { Mammography } \\
\text { features }\end{array}$} & \multicolumn{4}{|c|}{ Pathological type of carcinoma, $n$} & \multirow[b]{2}{*}{ Total, n (\%) } \\
\hline & Squamous & Spindle & Matrix-producing & Fibromatosis-like & \\
\hline Total & 7 & 3 & 2 & 1 & $13(100.0)$ \\
\hline \multicolumn{6}{|l|}{ Mass shape } \\
\hline Round & 1 & 0 & 1 & 0 & $2(15.4)$ \\
\hline Oval & 1 & 2 & 1 & 0 & $4(30.8)$ \\
\hline Lobular & 0 & 0 & 0 & 0 & $0(0.0)$ \\
\hline Irregular & 5 & 1 & 0 & 1 & $7(53.8)$ \\
\hline \multicolumn{6}{|l|}{ Mass margin } \\
\hline Circumscribed & 0 & 2 & 2 & 0 & $4(30.8)$ \\
\hline Microlobulated & 0 & 0 & 0 & 0 & $0(0.0)$ \\
\hline Indistinct & 4 & 1 & 0 & 0 & $5(38.5)$ \\
\hline Spiculated & 3 & 0 & 0 & 1 & $4(30.8)$ \\
\hline \multicolumn{6}{|l|}{ Mass density } \\
\hline High & 5 & 2 & 1 & 1 & $9(69.2)$ \\
\hline Iso & 2 & 1 & 1 & 0 & $4(30.8)$ \\
\hline Low & 0 & 0 & 0 & 0 & $0(0.0)$ \\
\hline \multicolumn{6}{|l|}{ Calcification } \\
\hline Present & 0 & 0 & 1 & 0 & $1(7.7)$ \\
\hline Not present & 7 & 3 & 1 & 1 & $12(92.3)$ \\
\hline
\end{tabular}
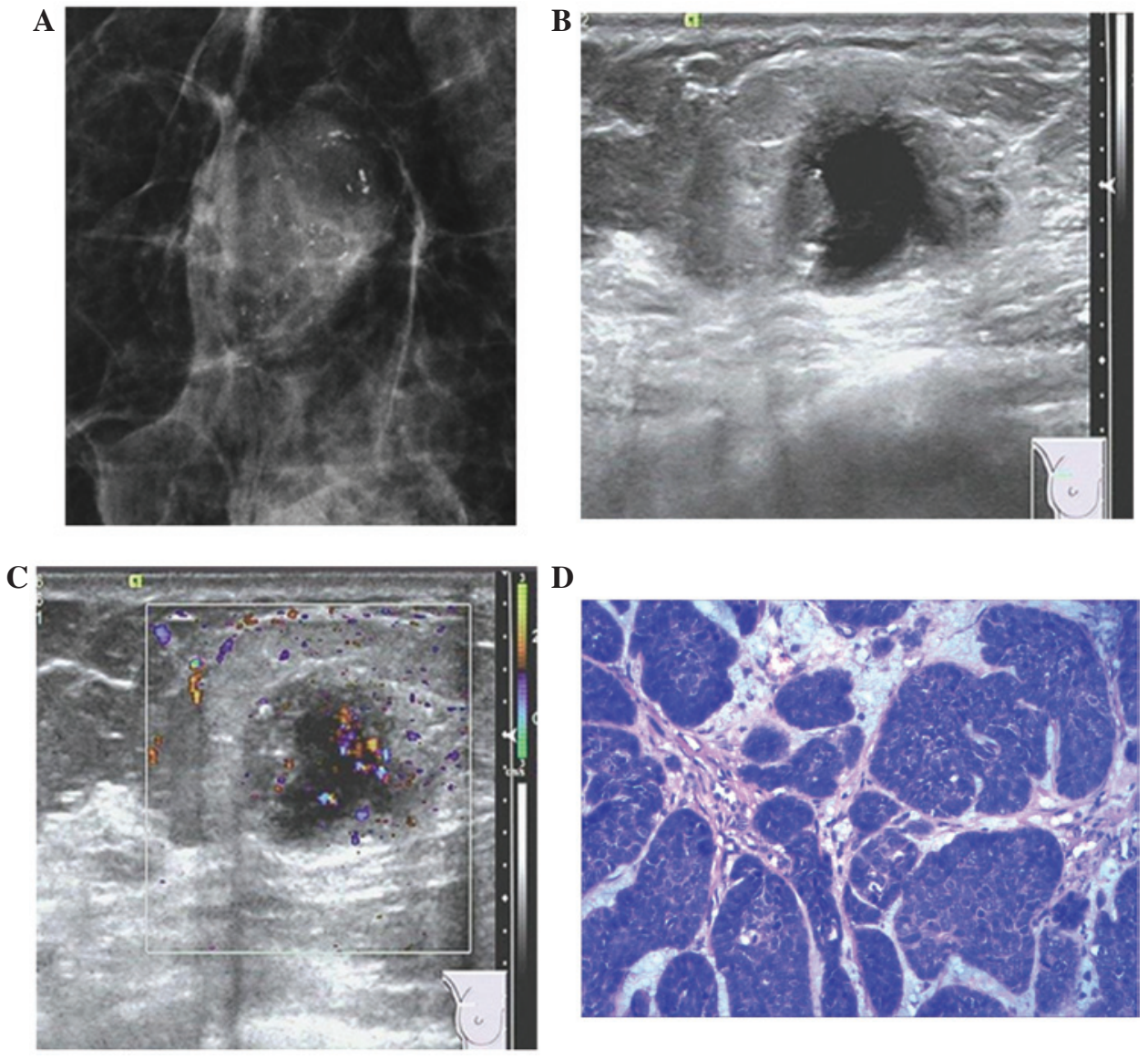

Figure 1. Representative imaging summary of metaplastic carcinoma of the breast. (A) Mediolateral-oblique mammogram reveals a circumscribed oval, slightly increased density mass with certain micro-calcifications. (B) Ultrasonogram reveals an irregular, mixed, solid and cystic mass with certain echogenical and posterior acoustic enhancement. (C) Color doppler ultrasonography demonstrates abundant blood flow in the mass. (D) Photomicrograph exhibits matrix-producing cell differentiation areas in the majority of the mass (hematoxylin-eosin staining; magnification, x200). Overall, the pathological diagnosis is a matrix-producing carcinoma. 
Table II. Sonography features and tumor types of 13 patients with breast metaplastic carcinomas.

\begin{tabular}{|c|c|c|c|c|c|}
\hline \multirow{2}{*}{$\begin{array}{l}\text { Sonography } \\
\text { features }\end{array}$} & \multicolumn{4}{|c|}{ Pathological type of carcinoma } & \multirow[b]{2}{*}{ Total, $\mathrm{n}(\%)$} \\
\hline & Squamous & Spindle & Matrix-producing & Fibromatosis-like & \\
\hline Total, $\mathrm{n}$ & 7 & 3 & 2 & 1 & $13(100.0)$ \\
\hline \multicolumn{6}{|l|}{ Mass shape } \\
\hline Round & 1 & 1 & 0 & 0 & $2(15.4)$ \\
\hline Oval & 0 & 1 & 1 & 0 & $2(15.4)$ \\
\hline Lobular & 0 & 0 & 0 & 0 & $0(0.0)$ \\
\hline Irregular & 6 & 1 & 1 & 1 & $9(69.2)$ \\
\hline \multicolumn{6}{|l|}{ Mass margin } \\
\hline Circumscribed & 3 & 2 & 1 & 0 & $6(46.2)$ \\
\hline Microlobulated & 0 & 0 & 0 & 0 & $0(0.0)$ \\
\hline Indistinct & 0 & 0 & 0 & 0 & $0(0.0)$ \\
\hline Spiculated & 4 & 1 & 1 & 1 & $7(53.8)$ \\
\hline \multicolumn{6}{|l|}{ Echogenicity } \\
\hline Homogeneous & 3 & 0 & 0 & 0 & $3(23.1)$ \\
\hline Complex & 4 & 3 & 2 & 1 & $10(76.9)$ \\
\hline \multicolumn{6}{|c|}{ Posterior acoustic enhancement } \\
\hline Present & 4 & 2 & 2 & 0 & $8(61.5)$ \\
\hline Not present & 3 & 1 & 0 & 1 & $5(38.5)$ \\
\hline
\end{tabular}

Table III. Immunohistochemical features and tumor types of 15 patients with breast metaplastic carcinomas.

\begin{tabular}{|c|c|c|c|c|c|c|c|c|c|}
\hline \multirow{3}{*}{$\begin{array}{l}\text { Pathological } \\
\text { type of carcinoma }\end{array}$} & \multirow[b]{3}{*}{ Total, $\mathrm{n}$} & \multicolumn{8}{|c|}{ Immunohistochemical markers } \\
\hline & & \multicolumn{2}{|c|}{ ER } & \multicolumn{2}{|c|}{ PR } & \multicolumn{2}{|c|}{ CerbB-2 } & \multicolumn{2}{|c|}{$\mathrm{P} 53^{\mathrm{a}}$} \\
\hline & & + & - & + & - & + & - & + & - \\
\hline Total, $\mathrm{n}$ & 15 & 2 & 13 & 1 & 14 & 1 & 14 & 7 & 4 \\
\hline Squamous & 8 & 2 & 6 & 1 & 7 & 1 & 7 & 5 & 3 \\
\hline Spindle & 3 & 0 & 3 & 0 & 3 & 0 & 3 & 0 & 0 \\
\hline Matrix-producing & 2 & 0 & 2 & 0 & 2 & 0 & 2 & 1 & 1 \\
\hline Fibromatosis-like & 1 & 0 & 1 & 0 & 1 & 0 & 1 & 0 & 0 \\
\hline Mixed metaplastic & 1 & 0 & 1 & 0 & 1 & 0 & 1 & 1 & 0 \\
\hline
\end{tabular}

${ }^{\mathrm{a} A s}$ the present study is retrospective, immunohistochemical information was only available for 11/15 patients. P53 expression was not indicated in 4/15 patients. ER, estrogen receptor; PR, progesterone receptor; CerB-2, receptor tyrosine-protein kinase erbB-2 (HER2/neu); P53, cellular tumor antigen p53; +, expressed; -, not expressed.

\section{Discussion}

Metaplastic carcinoma of the breast is a rare form of ductal carcinoma, which exhibits metaplastic transformation of glandular epithelium to non-glandular mesenchymal tissue (1-3). Numerous studies have reported that metaplastic carcinoma of the breast may originate from myoepithelial tissue (7). The carcinoma usually occurs among women aged $>50$ years, and often presents as a palpable mass that grows rapidly; therefore, the majority of patients present with larger palpable masses compared to invasive ductal carcinoma $(4,5)$.

According to the histological classification of breast tumors from the World Health Organization in 2012, metaplastic carcinoma of the breast may be classified into several types, as follows: Non-special type metaplastic carcinoma, including low-grade adenosquamous, fibromatosis-like, squamous cell and spindle cell carcinoma; metaplastic carcinoma with mesenchymal differentiation, including chondroid and osseous alteration; mixed metaplastic carcinoma; and myoepithelial cell carcinoma (8). Choi et al (4) reported that the most common type of metaplastic carcinoma was squamous cell carcinoma, which accounts for $0.5-3.7 \%$ of all types of breast cancer, followed by spindle cell carcinoma and matrix-producing carcinoma. The present study supports that squamous cell carcinoma is the most common type of metaplastic carcinoma, accounting for $53.8 \%$ of all cases, followed by spindle cell 
carcinoma at $20 \%$. These findings are consistent with previous studies $(4,5)$.

Metaplastic carcinoma exhibits various biological behaviors compared with invasive ductal carcinoma, and metaplastic carcinoma demonstrates a decreased tendency to involve the axillary lymph node (7). Leddy et al (1) reported that the incidence of axillary lymph node involvement ranges between 8 and $40 \%$, which is consistent with the findings of the present study, where axillary lymph node metastasis was observed in $6.7 \%$ of the patients. Metaplastic carcinoma tends to spread hematogenously to the lung and bone, rather than through the lymphatic system (3). There is not a large involvement of the axillary lymph node, despite the poor prognosis $(1,3,5)$.

Various subtypes of metaplastic carcinoma exhibit different imaging findings. Yang et al (5) reported that metaplastic carcinoma demonstrates more benign features compared with invasive ductal carcinoma, which often exhibits an oval or rounded shape, and the authors state that metaplastic carcinomas usually possess a circumscribed margin without malignant calcification in the mass. Leddy et al (1) revealed that metaplastic carcinomas may exhibit irregular shapes, indistinct or spiculated margins with certain amorphous or pleomorphic calcification. Previous studies have reported different views concerning the imaging findings of metaplastic carcinoma (4,5). Consequently, in the present study, the most common mammographic findings of squamous carcinoma were an irregular shaped mass with a spiculated margin and a high density, while spindle cell carcinoma and matrix-producing carcinoma exhibited an oval shaped mass with circumscribed margin and a slightly high density. On sonography, according to a previous study (9), metaplastic carcinoma usually demonstrates benign features with an oval or roundly shaped mass, a circumscribed margin and a homogeneous echogenicity with posterior acoustic enhancement. However, in the present study the most common sonographic features observed were a lobular or irregular shape, spiculated margin, heterogeneous echogenicity and posterior acoustic enhancement.

Therefore, the present results are not consistent with those of previous studies $(9,10)$. This may be due to the majority of patients in the present study possessing squamous cell carcinoma $(53.8 \%)$, the biological behavior of which is similar to that of invasive ductal carcinoma with invasive growth. Therefore, squamous cell carcinoma consistently demonstrates malignant features (3). In addition, since a fibrous capsule usually surrounds the mass of spindle cell carcinoma, spindle cell carcinoma consistently exhibits benign features (8). Therefore, the present study suggests that the imaging features of various subtypes of metaplastic carcinoma have certain characteristics. Few imaging features of metaplastic carcinoma of the breast have been reported, and since the number of patients reviewed in the present study is limited, additional investigation is required.

Metaplastic carcinoma demonstrates a poorer prognosis and lower survival rate compared with invasive ductal carcinoma (1). Esbah et al (2) revealed that the poor prognosis of metaplastic carcinoma may be explained by the increased tumor size, higher proliferation index, histopathological tumor heterogeneity, triple negative feature and lack of effective targeted therapies. Previous studies $(4,10,11)$ demonstrate that a more regular and more circumscribed mass may reflect a higher histological grade, while those lesions that appear benign may reflect tumor aggressiveness associated with a poorer prognosis and a low survival rate. This may be due to the association with the triple negativity of metaplastic carcinoma of the breast. Out of the 15 patients in the present study, only 2 patients expressed ER, 1 patient expressed PR and 1 patient expressed CerbB-2, which is similar to the results from previous studies $(1,4,8)$. The absence of hormone receptor expression on tumors restricts the application of hormonal therapy. Chao et al (12) reported no survival advantage of post-operative radiotherapy and various neoadjuvant chemotherapy regimens; however, different and/or mixed chemotherapy regimens and dose schedules may be more effective. Therefore, traditional surgery is the preferred treatment choice (2). However, Song et al (3) identified that local tumor recurrence was common following a simple mastectomy; therefore, surgical techniques, including radical mastectomy and modified radical mastectomy, such as axillary dissection, may improve the prognosis and survival rate of a patient. Additionally, local and distant metastases are more frequent than other types of breast cancer during the follow-up subsequent to surgery (1). Therefore, a systematic evaluation concerning local recurrence and surgical techniques is essential.

Diagnosing metaplastic carcinoma cannot rely on imaging features alone. Core needle biopsy and aspiration cytology may aid in a pre-operative diagnosis, but the probability of misdiagnosis would increase in the presence of hemorrhage or necrosis due to inadequate sampling or a poor choice of puncture region (1). In the majority of cases, the transition foci between metaplastic carcinoma and invasive ductal carcinoma were only observed following surgical biopsy $(1,3,9,11)$. Therefore, extensive sampling by surgical biopsy and immunohistochemical examination should be performed to avoid misdiagnosis.

In conclusion, metaplastic carcinoma is a rare form of breast cancer with various subtypes. It is important to differentiate metaplastic carcinoma from invasive ductal carcinoma, as the prognosis for metaplastic carcinoma is poor and the survival rate is low. Multimodality imaging examinations have certain imaging features, and combined with the observation of posterior acoustic enhancement and an absence of hormone receptor expression, may aid the differential diagnosis of metaplastic carcinoma and the development of a novel treatment strategy.

\section{References}

1. Leddy R, Irshad A, Rumboldt T, Cluver A, Campbell A and Ackerman S: Review of metaplastic carcinoma of the breast: Imaging findings and pathologic features. J Clin Imaging Sci 2: 21,2012 .

2. Esbah O, Turkoz FP, Turker I, Durnali A, Ekinci AS, Bal O, Sonmez OU, Budakoglu B, Arslan UY and Oksuzoglu B: Metaplastic breast carcinoma: Case series and review of the literature. Asian Pac J Cancer Prev 13: 4645-4649, 2012.

3. Song Y, Liu X, Zhang G, Song H, Ren Y, He X, Wang Y, Zhang J, Zhang Y, Sun S, et al: Unique clinicopathological features of metaplastic breast carcinoma compared with invasive ductal carcinoma and poor prognostic indicators. World J Surg Oncol 11: 129-138, 2013.

4. Choi BB and Shu KS: Metaplastic carcinoma of the breast: Multimodality imaging and histopathologic assessment. Acta Radiol 53: 5-11, 2012. 
5. Yang WT, Hennessy B, Broglio K, Mills C, Sneige N, Davis WG, Valero V, Hunt KK and Gilcrease MZ: Imaging differences in metaplastic and invasive ductal carcinomas of the breast. AJR Am J Roentgenol 189: 1288-1293, 2007.

6. Mercado CL. BI-RADS update. Radiol Clin North Am 52: 481-487, 2014

7. Pitts WC, Rojas VA, Gaffey MJ, Rouse RV, Esteban J, Frierson HF Kempson RL and Weiss LM: Carcinomas with metaplasia and sarcomas of the breast. Am J Clin Pathol 95: 623-632, 1991.

8. Shin HJ, Kim HH, Kim SM, Kim DB, Kim MJ, Gong G, Im SA and Cha ES: Imaging Features of Metaplastic Carcinoma with Chondroid Differentiation of the Breast. AJR 188:691-697, 2007.
9. Oberman HA: Metaplastic carcinoma of the breast. A clinicopathologic study of 29 patients. Am J Surg Pathol 11: 918-929, 1987.

10. Park JM, Han BK, Moon WK, Choe YH, Ahn SH and Gong G: Metaplastic carcinoma of the breast: Mammographic and sonographic findings. J Clin Ultrasound 28: 179-186, 2000.

11. Kaas R, Kroger R, Hendriks JH, Besnard AP, Koops W, Pameijer FA, Prevoo W, Loo CE and Muller SH: The significance of circumscribed malignant mammographic masses in the surveillance of BRCA 1/2 gene mutation carriers. Eur Radiol 14: 1647-1653, 2004.

12. Chao TC, Wang CS, Chen SC and Chen MF: Metaplastic carcinomas of the breast. J Surg Oncol 71: 220-225, 1999. 